\title{
Diagnóstico del estado actual del flujo de información entre el destino La Habana y los visitantes extranjeros
}

\author{
Diagnosis of the current status of the information flow between Havana \\ destination and foreign visitors \\ Daikel Adriano Abreu Rodríguez. ${ }^{1}$, Sergio Valdivia Izquierdo ${ }^{2} \&$ Yennifer Sánchez \\ Borges. $^{3}$
}

\begin{abstract}
The information to the visitors is a key aspect in the management of touristic destinations, since the available information for both current and potential clients can determine the choices regarding to travel to a specific destination and which activities to do during the stay. The current research is related to such subject and his objective is to do a diagnostic of the present situation of the flow of information between destination and client in Havana. This study is part of a scientific and technical service from the Tourism Faculty of the University of Havana to the National Touristic Information Office. The accomplishment of the proposed objective was determined by realization of interviews to infomediaries and surveys to visitors. It allowed obtaining as main results: the knowledge about the information that the visitors request to the infomediaries and how these last ones provide it, as well as a description of the visitors of Havana according to the information sources for the organization of their own travels.
\end{abstract}

Key Words: Touristic Infomediaries, Visitors, Touristic Information, Information Sources.

\section{Resumen}

La información al visitante es un elemento clave en la gestión de destinos turísticos, la información de que dispongan los clientes actuales y potenciales puede determinar la decisión de viajar a un destino específico y las actividades a realizar en el mismo. La investigación que se presenta aborda precisamente este tema y persigue como objetivo

\footnotetext{
${ }^{1}$ MSc. / Facultad de Turismo, Universidad de La Habana / daikeladriano@gmail.com

${ }^{2}$ Lic. / Facultad de Turismo, Universidad de La Habana / sergiovaldiviaizq@gmail.com

${ }^{3}$ Lic. / Facultad de Turismo, Universidad de La Habana / ysanchezb97@gmail.com
} 
diagnosticar la situación actual del flujo de información destino - cliente para La Habana. El estudio forma parte de un servicio científico - técnico que realiza la Facultad de Turismo de la Universidad de La Habana para la Oficina Nacional de Información Turística. El logro del objetivo propuesto estuvo determinado por la realización de entrevistas a infomediarios y de encuestas a visitantes. Lo cual permitió alcanzar como principales resultados: el conocimiento sobre la información que solicitan los visitantes a los infomediarios y cómo estos últimos logran brindarla, así como una caracterización de los clientes de La Habana en cuanto a las vías utilizadas para la obtención de la información en la organización y realización del viaje.

Palabras Clave: Infomediario Turístico, Visitantes, Información Turística, Vías de obtención de información

\section{Introducción}

La información turística sobre un destino es un elemento clave para la organización y realización del viaje por parte de los visitantes. Todos los destinos turísticos y específicamente las entidades que los gestionan debían tener entre sus prioridades la emisión de información sobre el mismo a los visitantes reales y potenciales.

La información existente sobre un destino determina en la selección de este para viajar, determina en la decisión de viajar a un sitio o no, e influye determinantemente en la organización del viaje. Asimismo, a partir de la información con qué cuenta el cliente, toma decisiones con relación a la duración de la estancia, con relación al alojamiento a utilizar y entre otros con relación a las actividades a realizar.

Martín (2015) afirma:

Posiblemente ningún otro sector de la economía tenga tanta necesidad como el turismo de que el cliente disponga de buena información para que compre y, con ello, alcanzar sus objetivos de ingresos y beneficios. Es que los viajes se compran antes de ejecutarse y el "consumo turístico" es muy lejos del lugar de compra. Como el cliente no puede inspeccionar físicamente el lugar a visitar, requiere de informaciones oportunas y exactas de lo que está comprando (p. 99).

Es por ello que en la actual gestión de destinos se comprende la información al visitante como uno de los elementos siempre presentes en las funciones de las entidades gestoras. Esta información al visitante está enfocada según el nivel de desarrollo del destino y el nivel de complejidad que ha alcanzado su gestión como un grupo de informaciones ofrecidas a los clientes o como un sistema de información al visitante o como un subsistema dentro del sistema de gestión del destino. 
Países europeos como Francia, España y Holanda tienen un gran desarrollo en la información al visitante, es aquí donde surgen las Oficinas de Información Turística. Las cuales tienen como objetivo gestionar servicios relacionados con la atención, orientación y asesoramiento a los visitantes que llegan al destino (Valdés, Martín, Aza, Del Valle y Delgado, 2011).

La realidad actual, determinada por el amplio desarrollo de las tecnologías de la información y las comunicaciones ha obligado a estas oficinas de información turística a evolucionar y ofrecer la información no sólo en el destino sino también a través de las páginas webs que los clientes pueden revisar, personalmente, desde el origen, antes de la realización del viaje.

Moratilla et al. (2004, citado por Valdés et al. 2011) expresa:

De esta forma y con el desarrollo de las Tecnologías de la Informática y las Comunicaciones, TICs, comienzan a surgir en Internet los primeros sistemas de información al visitante que, al principio fueron pequeños portales a modo de prototipo para probar nuevas vías de comunicación con turistas potenciales, que permitiesen contribuir al crecimiento turístico de determinadas zonas, generalmente rurales (p. 26).

No obstante, reconocer que, la información que obtienen los visitantes reales y potenciales sobre el destino no depende únicamente, o no es únicamente la información emitida por las entidades que lo gestionan, es importante a la hora de definir cómo se organizará el flujo de información hacia el cliente. Martín y Abreu (2013) diseñaron un modelo que explica y grafica la manera en que fluye la información sobre un destino hacia los visitantes reales y potenciales.

La figura 1 refleja una adaptación y actualización hecha por los autores de esta investigación a partir de la propuesta original referenciada en el párrafo anterior. En esta actualización se incluyen nuevos infomediarios que no habían sido considerados originalmente. Además, se omite la consideración del momento en que cada infomediario participa en el ofrecimiento de información al cliente (antes o durante el viaje). Esta última modificación se hace teniendo en cuenta precisamente el alto desarrollo de la TICs, ya mencionado. No se puede decir que las entidades externas al destino, aunque ofrecen la mayor parte de la información antes del viaje, no brindan información a los viajeros durante su estancia en el destino a través de páginas webs, aplicaciones de teléfonos móviles, vía telefónica, asistentes de viajes, entre otros. Igualmente, las entidades propias del destino, aunque brindan la mayor parte de la información durante la realización del viaje, pueden brindar la información a los clientes a través de páginas webs, correo electrónico, etc.

Es importante, tanto en el diagrama original como en el que se presenta ahora, el término infomediario turístico. Entendido este como toda aquella entidad o persona que media entre el destino y el visitante (real o potencial) brindando o transmitiendo información 
(Martín y Abreu, 2013). Todo el que transmita información sobre el destino a un cliente es un infomediario turístico para ese destino. Como se había dicho antes, la información que se brinda, puede determinar la decisión de compra y otros aspectos importantes en la organización del viaje, por lo cual, los gestores del destino deben ocuparse de influir y monitorear la información ofrecida por todos los infomediarios, aunque no puedan controlar los contenidos emitidos por muchos de ellos.

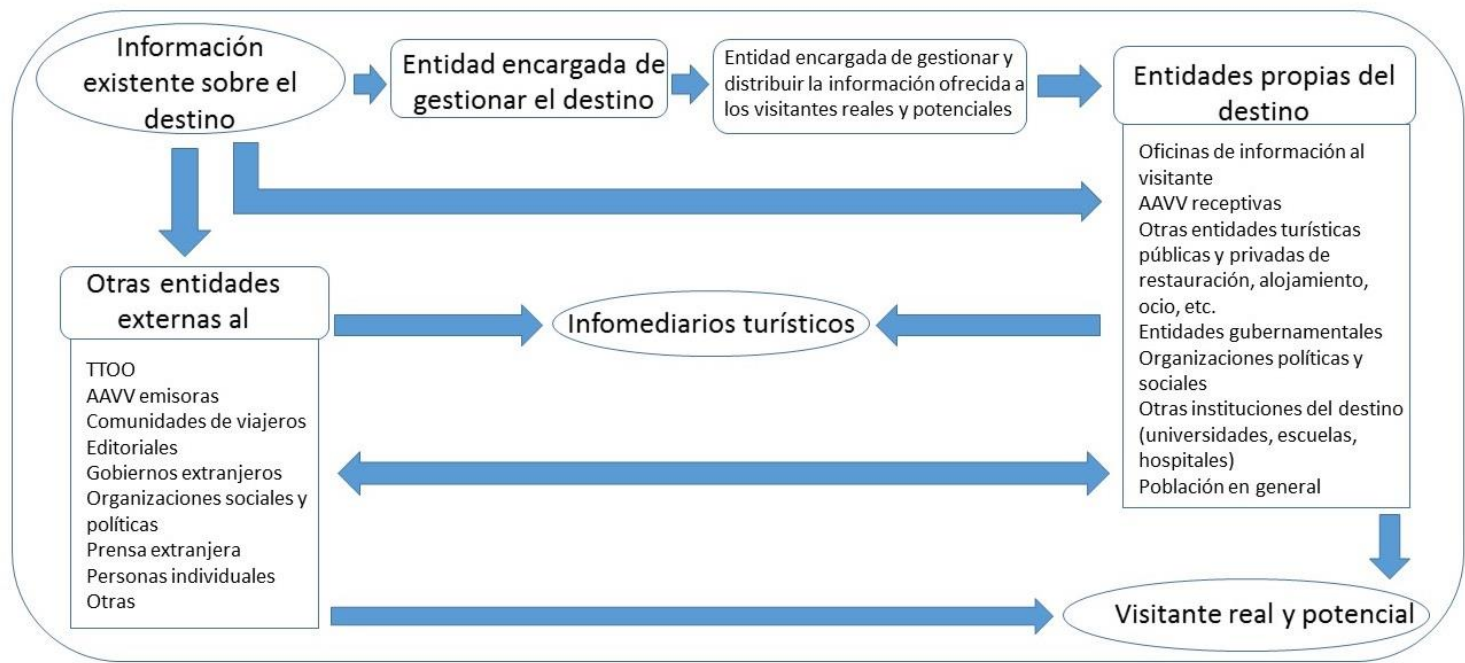

Figura 1. Diagrama de flujo de información destino - cliente. Elaboración propia a partir de la propuesta inicial hecha por Martín y Abreu (2013).

Fuente: Martín y Abreu (2013).

Se recoge a su vez en el esquema la idea de que tanto los infomediarios externos al destino como los propios del destino se influyen mutuamente. Usan la información publicada por los otros y, la organizan y presentan utilizando criterios propios. Esto es inevitable que ocurra, pero los gestores del destino pueden, a través de acciones concretas, influir en la información que ofrecen las entidades externas a él.

En Cuba el encargado de gestionar el destino país y los destinos específicos es el Ministerio de Turismo con sus delegaciones provinciales. Asimismo, la Oficina Nacional de Información Turística con los centros de información turística, es la encargada de gestionar la información que se ofrece a los visitantes reales y potenciales del destino (MINTUR, 2019).

La Oficina Nacional de Información Turística gestiona, por tanto, el Sistema de Información al Visitante (SIV) (Castellanos, 2014). El SIV contempla como procesos la captación, manejo, evaluación, análisis, síntesis, composición, almacenamiento, traducción, empaquetamiento y distribución de la información (Oficina Nacional de Información Turística, 2014).

Cuba cuenta para el desarrollo de esta actividad y para cumplir el objeto social de la Oficina Nacional de Información Turística con 37 centros de información al visitante 
distribuidos en 13 provincias del país, de ellos 9 se encuentran en aeropuertos (Manzano, 2016). Estos centros de información están subordinados metodológicamente a la oficina nacional, pero actualmente, se subordinan administrativamente a las delegaciones provinciales del Ministerio de Turismo de la República de Cuba.

A partir de estos cambios administrativos y teniendo en cuenta los cambios más recientes en las tecnologías de la información (la masificación de muchas de ellas), la Oficina Nacional de Información Turística se encuentra en el proceso de actualización del Sistema de Información al Visitante. Este proceso de actualización ha llevado a los directivos de la oficina a solicitar a la Facultad de Turismo de la Universidad de La Habana un servicio científico - técnico que contribuya al logro de este propósito.

Uno de los puntos álgidos que está dentro de este proceso de actualización es precisamente la retroalimentación. Es decir, tener un esquema funcional, que permita en tiempo real, saber la información que están recibiendo los clientes sobre el destino Cuba y sus destinos específicos y cuáles son las necesidades insatisfechas de información; es importante para la implementación del nuevo sistema.

Como parte del servicio científico - técnico a realizar se debe diseñar el subsistema de retroalimentación y para poder hacerlo se debe conocer el estado actual del tema. Por tanto, el estudio que se expone a continuación persigue como objetivo: Diagnosticar el estado actual del flujo de información destino - cliente. Y se usa como caso de estudio el destino La Habana.

\section{Desarrollo:}

\section{Cuestiones de diseño de la investigación}

Para el desarrollo del diagnóstico se realizaron entrevistas a infomediarios y encuestas a visitantes del destino La Habana. Cada de una de las técnicas aplicadas perseguían diferentes objetivos, pero ambas de conjunto permitieron lograr el objetivo del estudio, expuesto en el acápite anterior.

\section{Entrevista a infomediarios}

Las entrevistas se realizaron en las 4 zonas turísticas de La Habana: Miramar, Vedado, Centro Histórico y Este de la ciudad. Fueron aplicadas por los estudiantes de 2do año de la Licenciatura en Turismo de la Universidad de La Habana, durante el desarrollo de sus prácticas pre-profesionales entre el 18 de enero y el 8 de febrero del año 2019. Los 90 estudiantes fueron distribuidos por las 4 zonas en proporción con la cantidad de negocios existentes y el flujo diario de visitantes extranjeros.

Para evitar que coincidieran varios estudiantes en los mismos lugares y que visitaran los mismos negocios, se dividieron en equipos distribuidos por calles y/o zonas específicas. El criterio para seleccionar a los entrevistados estuvo determinado por el hecho de ser 
personal de contacto en cualquier de los negocios turísticos que atienden visitantes extranjeros habitualmente.

El guion de la entrevista incluía 6 preguntas de contenido y otras dos preguntas sobre el negocio y la persona que brindaba la información respectivamente. Los objetivos de la entrevista fueron: 1- determinar la información que solicitaban los clientes en las diferentes entidades turísticas y 2- Identificar de dónde obtenía la información el personal de contacto que se relacionaba con los clientes y le brindaba la información solicitada. En total fueron realizadas 1234 entrevistas en el período de tiempo y en las zonas mencionadas anteriormente.

\section{Encuesta a visitantes}

Para la realización de la encuesta, los cuestionarios (en idiomas inglés y español) fueron aplicados igualmente por los estudiantes de 2do año del Curso Diurno de la Facultad de Turismo y en las 4 zonas turísticas mencionadas anteriormente. Asimismo, se dividieron los estudiantes por zonas y/o calles específicas para evitar la concentración de ellos en el mismo lugar y evitar que varios de ellos abordaran al mismo visitante. La ejecución de esta tarea se estuvo realizando entre el 22 de febrero y el 2 de marzo del mismo año y se logró encuestar a 1956 individuos.

El cuestionario aplicado comprendía 7 preguntas de contenido y una pregunta destinada a caracterizar los sujetos que formaron parte de la muestra. La encuesta tenía como objetivos: 1- Determinar las vías por las que los visitantes obtienen información sobre el destino antes y durante la realización del viaje y 2-Identificar las relaciones que existen entre las distintas vías que los visitantes utilizan para informarse y sus características sociodemográficas.

En la tabla 1 se muestran los parámetros utilizados para el cálculo de la muestra, así como la muestra planificada y la que realmente formó parte del estudio. El tamaño real de la muestra supera significativamente al tamaño previsto inicialmente y por tanto reduce el margen de error y brinda mayor solidez a los resultados obtenidos. Para la selección de los sujetos que formaron parte de la muestra se tuvo en cuenta que se encontraran en alguna de las zonas turísticas mencionadas anteriormente, que fueran visitantes extranjeros y que estuvieran dispuestos a formar parte del estudio. 
Tabla 1: Parámetros utilizados para el cálculo de la muestra.

\begin{tabular}{lr}
\hline \multicolumn{2}{c}{ Parámetros utilizados para el cálculo de la muestra } \\
\hline Estimación de visitantes a & 2419561 \\
La Habana en el año 2019 & 2419561 \\
Población utilizada & $95,00 \%$ \\
Nivel de confianza & 1,96 \\
K & 0,5 \\
p & 0,5 \\
q & 0,022158 \\
\hline
\end{tabular}

Nota: Elaboración propia a partir de los cuestionarios aplicados.

Para el cálculo de la muestra se usó como población a la cantidad supuesta de visitantes que tendría La Habana en el año 2019. Debido a que no son públicas en este momento las cifras de visitantes al destino (La Habana), ni siquiera en alguno de los últimos 10 años, fue necesario estimar el número de visitantes usando como base la cifra de 1126144 del 2008 (Pardo, 2009). Se calculó la tasa de crecimiento anual del destino país Cuba del año 2008 al 2018 y se extrapoló esa tasa de crecimiento al destino La Habana, permitiendo así estimar los visitantes para el 2019. Los datos de visitantes del país en el 2008 y 2018 fueron extraídos de la web oficial de la Oficina Nacional de Estadísticas e Información (ONE, 2011 y ONEI, 2019).

\section{Información obtenida mediante la aplicación de las técnicas}

\section{Entrevista a infomediarios}

Las entrevistas realizadas abarcaron una representación bastante amplia de los infomediarios existentes en el destino. Se realizaron entrevistas en restaurantes privados y estatales, hoteles administrados por el estado y por empresas extranjeras y casas de rentas administradas por privados. Asimismo, también fueron entrevistadas personas en tiendas de la empresa Caracol, kioscos de artesanías, burós de ventas de las agencias de viajes, entre otros. En estas entidades se entrevistaron a dependientes gastronómicos, dependientes de tiendas, cantineros, recepcionistas, responsables de relaciones públicas, etc. y en el caso de los alojamientos privados a los administradores y/o propietarios.

La primera cuestión (pregunta 1 del guion) abordada en la entrevista se refería a si los clientes solicitaban información sobre el destino no relacionada específicamente con el negocio en cuestión. La mayoría de los entrevistados respondieron afirmativamente a esta cuestión y además explicaron las informaciones que eran solicitadas, que era sobre lo que se refería la segunda pregunta.

Las informaciones que son más solicitadas por los visitantes (pregunta 2 del guion), según los entrevistados, son sobre: la Wi-Fi y conexión a internet, direcciones (cómo llegar a determinado lugar), cambio de monedas y ubicación de los bancos, transporte (taxis y 
otros alternativos), precios y horarios de restaurantes y museos y sobre centros nocturnos (bares y discotecas) a los que pudiesen ir. Además, preguntan sobre lugares interesantes a visitar en el país, sobre la cultura del pueblo, sobre la política y la sociedad, sobre sitios históricos y sobre lugares donde puedan conocer la música y la danza tradicionales de Cuba.

Al cuestionar a los entrevistados sobre la frecuencia con que los visitantes solicitan información (pregunta 3 del guion), respondieron que era bastante habitual, aunque no todos los clientes preguntan, ellos sí atienden diariamente a personas que piden información. Los infomediarios destacan en este aspecto que la barrera idiomática influye en la comunicación y el intercambio con los turistas, cuando no pueden comunicarse bien porque no dominan algún idioma común, el volumen de información solicitada es mucho menor.

La mayoría de los entrevistados son capaces de brindar la información que solicitan los visitantes (pregunta 4 del guion), no obstante, hay un grupo de ellos que no siempre pueden responder a las preguntas realizadas. Este último grupo de infomediarios explica que ello se debe a que en ocasiones desconocen sobre lo que los clientes están preguntando y en otras ocasiones por cuestiones relativas a las barreras idiomáticas, ya mencionadas anteriormente.

Sobre las fuentes de donde los entrevistados obtienen la información brindada a los visitantes (pregunta 5 del guion) las respuestas son muy diversas. Algunos utilizan internet, otros se basan en su experiencia cotidiana, otros consultan aplicaciones de teléfonos móviles (ConoceCuba, TripAdvisor, AlaMesa, CubaPaladar, HabanaTrans, entre otras), otros se nutren de los medios de comunicación masiva del país (radio, televisión, periódicos, revistas) y otros utilizan las redes sociales "físicas" (redes de amigos y conocidos) y de internet.

Para muchos de los entrevistados sería de gran ayuda recibir información actualizada por parte de los gestores del destino (pregunta 6 del guion), pero hay otros que tienen criterios diferentes. La minoría considera que está bastante actualizado y que poseen toda la información necesaria. No obstante, la mayoría de los infomediarios creen que sería de gran utilidad recibir información actualizada, argumentan que, en el destino surgen y se modifican lugares a diario, además, consideran que poseer información sobre el destino en general le aporta valor a su negocio y logran una mayor satisfacción de sus clientes. Importante destacar las respuestas de algunos que además de estar dispuestos a recibir información, ya utilizan los materiales (guías y mapas) elaborados por la Oficina Nacional de Información Turística.

\section{Encuesta a visitantes}

Los visitantes encuestados representan a 63 nacionalidades diferentes, dentro de las que se encuentran los principales mercados del destino. Además, la muestra está conformada 
por clientes de ambos sexos (55\% masculino y $45 \%$ femenino) y por un rango de edad que va desde los 18 hasta los 83 años.

Del total de los visitantes que formaron parte de la muestra el 57,9\% se encontraban realizando su primera visita a la Isla. Dentro de los restantes encuestados que habían estado con anterioridad en el destino lo más común es que hayan estado en Cuba de 2 a 4 veces antes, representando el $51 \%$ dentro de los que no estaban realizando la primera visita.

Las vías por las cuáles los clientes conocen sobre Cuba antes de su primera visita (pregunta 2 del cuestionario y de selección múltiple) son varias, no obstante, vale la pena señalar cuatro de ellas que son las que mayormente han sido seleccionadas por los entrevistados: 1- Amigos y familiares, 2- Páginas webs sobre Cuba, 3- Redes sociales en internet y 4- Cultura general (ver tabla 2). Los que marcaron otra vía, conocieron sobre Cuba por trabajo, porque nacieron en el país o porque lo sorprendieron con el viaje como regalo.

Tabla 2: Vías por las que conocen sobre Cuba antes de la primera visita.

\begin{tabular}{lrr}
\hline \multicolumn{3}{c}{ Antes de la 1ra visita conoció sobre Cuba por: } \\
\hline & 869 & 44,4 \\
\hline Cultura General & 1001 & 51,2 \\
Amigos y/o Familiares & 285 & 14,6 \\
Revistas o Periódicos & 354 & 18,1 \\
Radio o Televisión & 749 & 38,3 \\
Agencias de Viajes & 916 & 46,8 \\
Internet: Redes Sociales & 935 & 47,8 \\
Internet: Páginas sobre Cuba & 273 & 14 \\
Internet: Blogs Comunitarios & 243 & 12,4 \\
Internet: Otras páginas webs & 137 & 7 \\
Organización académica o & 30 & 1,5 \\
social & & \\
Otra vía & &
\end{tabular}

Nota: Elaboración propia a partir de los cuestionarios aplicados.

Es muy interesante el hecho de que cuando comparamos las respuestas seleccionadas entre los visitantes que viajan por primera vez con los repitentes, proporcionalmente tienen el mismo comportamiento (ver figura 2). Esto permite afirmar que a pesar del desarrollo sostenido que tienen las tecnologías de la información, la manera en que los clientes conocen sobre Cuba ha mantenido la misma estructura en los últimos años. Sólo hay una ligera disminución en los indicadores: Amigos y/o Familiares y Revistas o Periódicos, el resto de los indicadores se mantienen casi idénticos. 


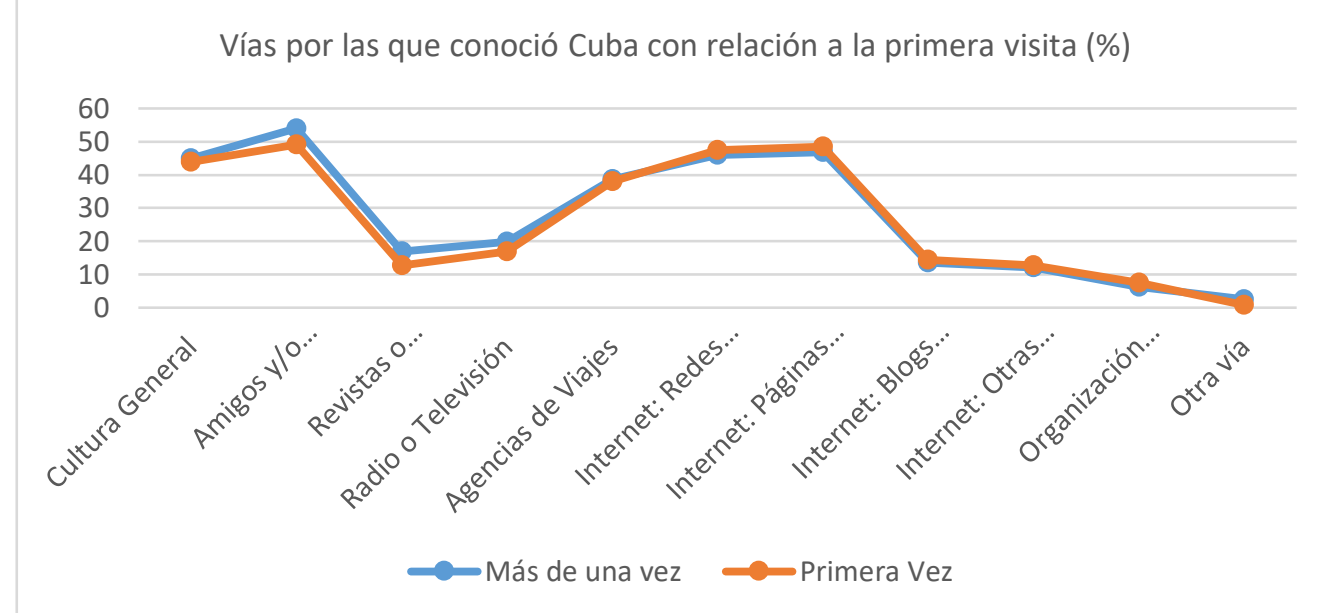

Figura 2. Relación entre las vías por las cuales conoció sobre Cuba y la condición de repitente o primerizo del encuestado. Elaboración propia a partir de los cuestionarios aplicados.

Cuando se compara el comportamiento por sexo en cuanto a la variable anteriormente mencionada no hay diferencias entre los dos grupos, sólo el sexo femenino supera ligeramente al masculino en el indicador Amigos y/o Familiares, en el resto de los indicadores el comportamiento es idéntico. Sin embargo, cuando se hace este mismo análisis con relación a las edades (ver figura 3), sí se encuentran diferencias, sobre las cuales vale la pena llamar la atención. La primera de ellas es que disminuye la frecuencia de selección del indicador Cultura General de conjunto con la edad. La segunda es que el indicador Amigos y/o Familiares es seleccionado por un porciento significativo dentro de cada grupo etario, a pesar de que es menos utilizados por los mayores de 70 años. El indicador Revistas o Periódicos crece casi de la misma manera que la edad y el indicador Radio y Televisión es seleccionado de la misma manera por los encuestados sin importar su edad. Por último, llamar la atención en que los más adultos utilizaron más las agencias de viajes para enterarse de Cuba y en que los más jóvenes supieron de la existencia de la Isla por las diferentes vías existentes en Internet mucho más que los más adultos. 


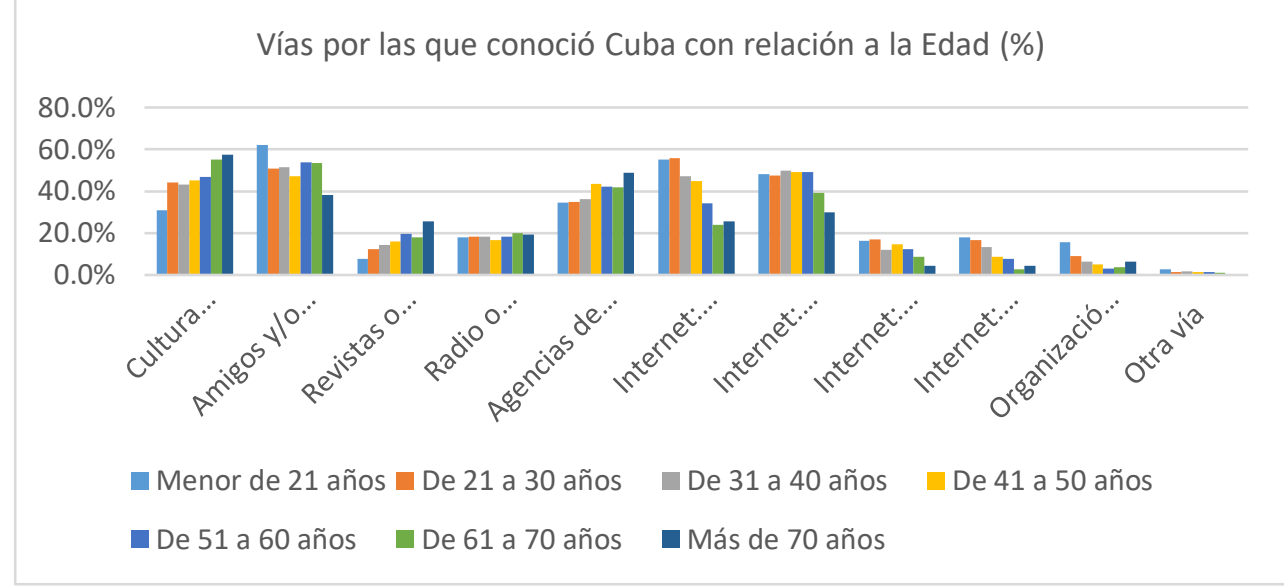

Figura 3. Relación entre las vías por las cuales conoció sobre Cuba y la Edad. Elaboración propia a partir de los cuestionarios aplicados.

La siguiente variable está referida a las vías por las cuales los visitantes obtienen la información necesaria para la organización del viaje (pregunta 4 del cuestionario y de selección múltiple). Destacan como las más seleccionadas por los encuestados: Páginas sobre Cuba en Internet (48,9\%), Amigos y familiares (46.1\%), Agentes de viajes (43.5\%), Redes sociales en Internet (42.6\%) y Guías turísticas (libros) (40.0\%). (ver tabla 3).

Tabla 3: Vías por las cuales obtienen información para la organización del actual viaje.

Vías por las cuales se obtuvo la información para la organización del actual viaje

\begin{tabular}{lrc}
\hline & Frecuencia & \% \\
\hline Experiencia personal de viajes anteriores & 657 & 33,6 \\
Amigos y/o Familiares & 902 & 46,1 \\
Agentes de Viajes & 851 & 43,5 \\
Guías Turísticas (libros) & 782 & 40,0 \\
Internet: Redes Sociales & 834 & 42,6 \\
Internet: Páginas sobre Cuba & 957 & 48,9 \\
Internet: Blogs comunitarios & 316 & 16,2 \\
Internet: Otras páginas webs & 193 & 9,9 \\
Otra vía & 26 & 1,3 \\
\hline
\end{tabular}

Nota: Elaboración propia a partir de los cuestionarios aplicados.

Los visitantes que seleccionaron "Otra vía" obtuvieron la información a través de sus centros de estudios (escuelas y universidades), a través de la iglesia, a través de sus centros de trabajo y viendo películas y videos sobre Cuba. Asimismo, hubo un grupo de informaciones que algunos encuestados (10.8\%) refirieron no haber conseguido durante la organización del viaje (pregunta 5 del cuestionario y abierta), dentro de ellas las más recurrentes son: detalles sobre el cambio de monedas, explicación de los servicios que 
son en CUP y los que son en CUC y explicación del funcionamiento del transporte y los precios de los taxis y la transportación nacional.

$\mathrm{Al}$ relacionar las dos variables que se observan en la figura 4, se pueden arribar a varias conclusiones que, aunque puedan parecer obvias algunas de ellas, es significativo haber podido demostrarlas con el estudio. Los visitantes se apoyan para la organización de su viaje en las informaciones brindadas por los familiares y amigos, sin importar la vía utilizada para su organización. No obstante, los que organizan su viaje de forma independiente o por otra vía diferente a las propuestas en el cuestionario, lo hacen con mayor frecuencia. Es muy notable que la utilización de los agentes de viajes para informarse en la organización del viaje está determinada por la manera en que este se organiza, los visitantes que utilizaron las agencias de viajes para la organización de su viaje, también la utilizaron para obtener la información necesaria en su organización. Se debe destacar, que mientras más se deposita la responsabilidad en una misma agencia, más la información obtenida depende de esta. Es relevante también, que los que organizaron el viaje de forma independiente o por otra vía distinta a las mencionadas utilizaron en muy poca medida los agentes de viajes para informarse.

Los visitantes agrupados por las formas en que organizan el viaje utilizan más o menos igual las diferentes vías de información por internet y es notable como los que organizan el viaje por otra vía diferente, también se informan para la organización del viaje por otra vía diferente. Se trata de los que organizan sus viajes a través de escuelas, centros de trabajo e iglesias, que también reciben la información necesaria para la organización del viaje a través de estas instituciones.

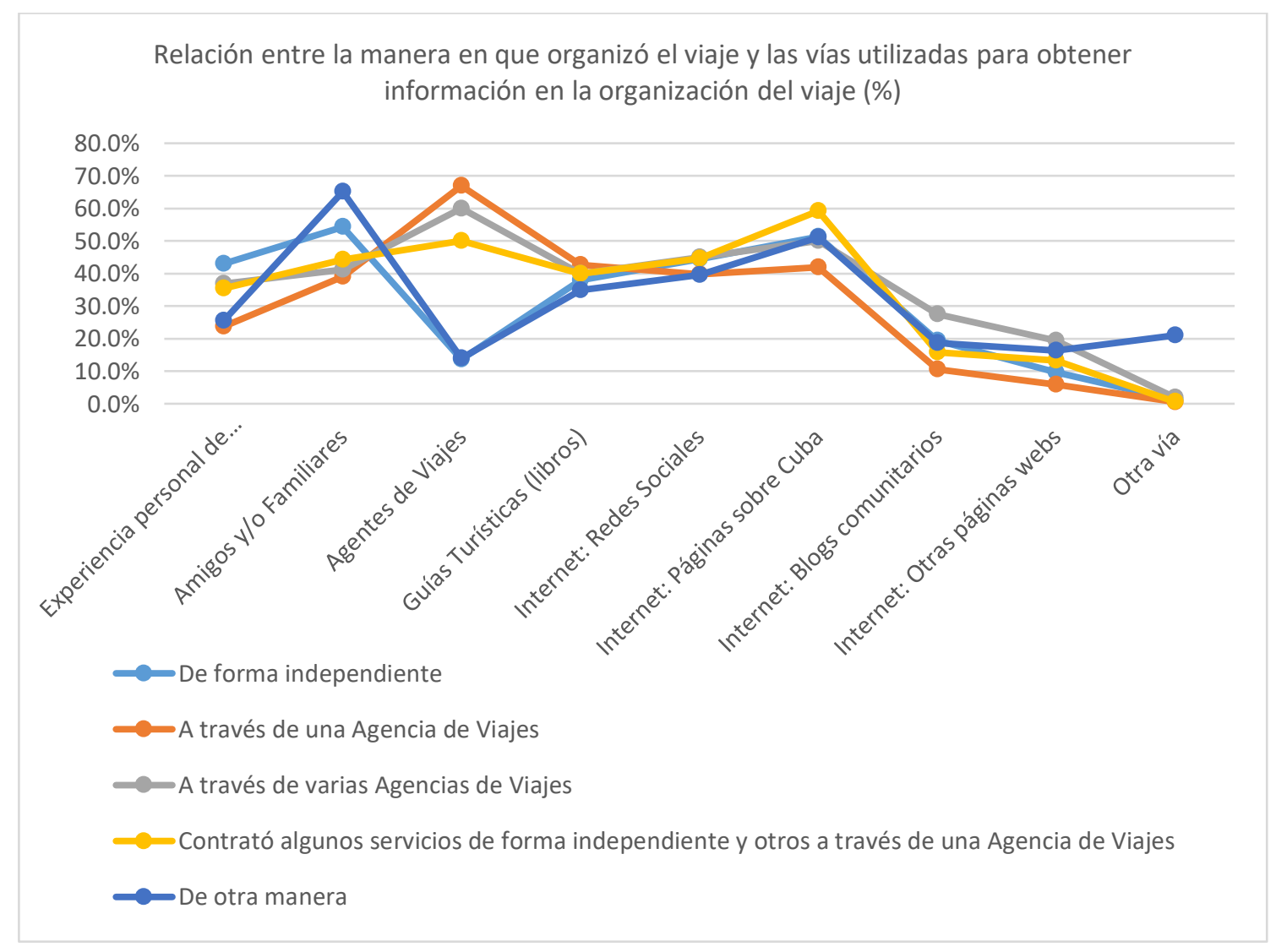


Figura 4: Relación entre la manera que los visitantes organizan su viaje y las vías que utilizan para obtener la información necesaria en la organización del viaje. Elaboración propia a partir de los cuestionarios aplicados.

Al analizar la variable con relación al sexo el comportamiento es muy similar entre ambos sexos, solamente se pueden señalar dos ligeras diferencias: los visitantes de sexo masculino se basan más en las experiencias de viajes anteriores y los visitantes de sexo femenino se basan más en los criterios de familiares y amigos. Sin embargo, en cuanto a la edad hay algunas diferencias sobre las cuales se debe llamar la atención.

Los más jóvenes son los que más utilizan la información brindada por los familiares y amigos para organizar sus viajes. Los más adultos son los que más utilizan las informaciones brindadas por los agentes viajes y los más jóvenes son los que menos se basan en esta vía de información para la organización de su viaje. Nuevamente, son los más jóvenes los que más utilizan las distintas vías existentes en internet para obtener información y en especial las redes sociales.

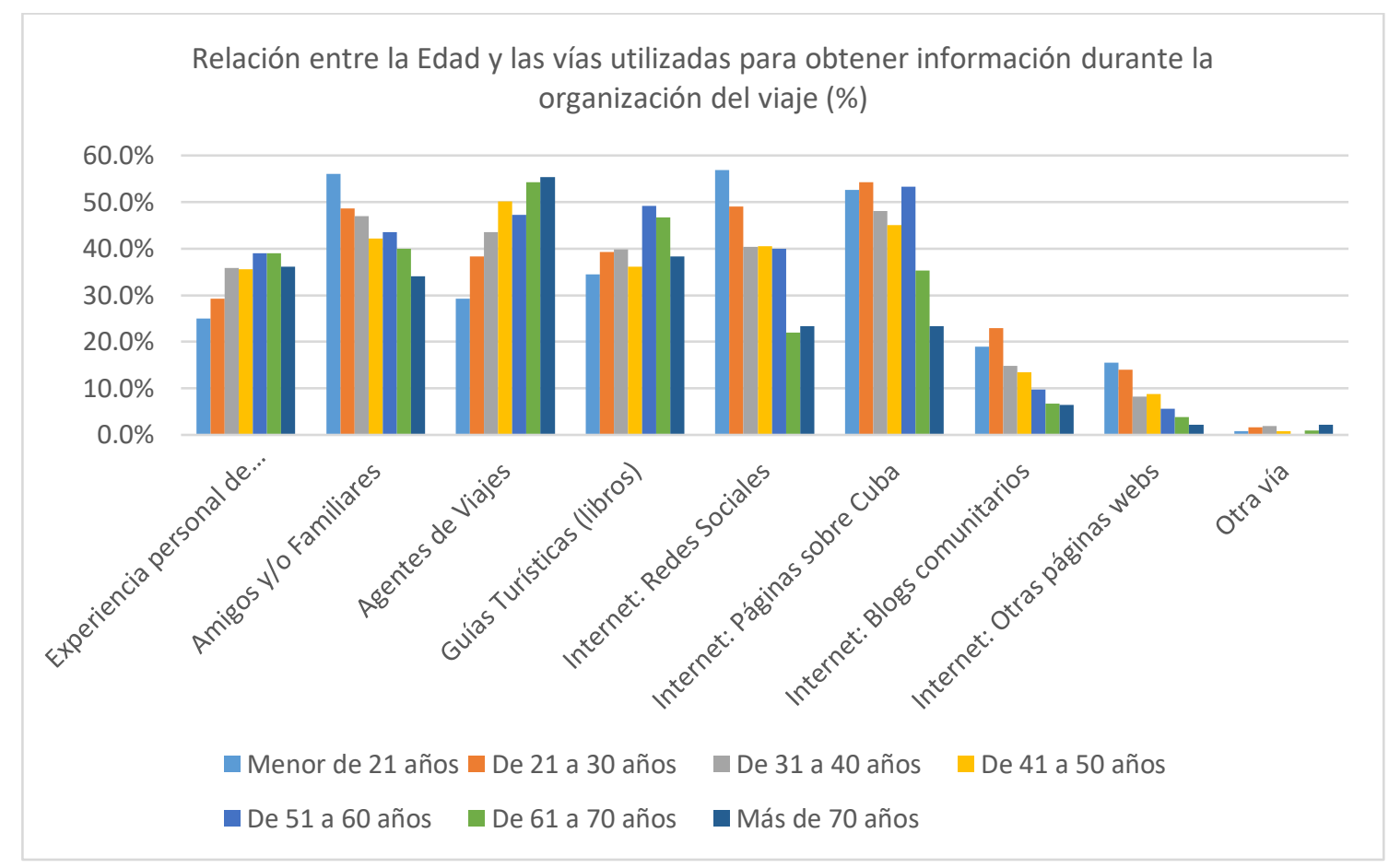

Figura 5: Relación entre la Edad y las vías que utilizan para obtener la información necesaria en la organización del viaje. Elaboración propia a partir de los cuestionarios aplicados. 
Tabla 4: Vías por las cuales obtienen información durante la realización del viaje.

Vías por las cuales se obtuvo la información durante la realización del actual viaje

\begin{tabular}{lrc}
\hline & Frecuencia & \% \\
\hline Amigos y/o Familiares & 523 & 26,7 \\
Preguntando en la calle & 1016 & 51,9 \\
Agentes de viajes o guías turísticos & 815 & 41,7 \\
Mapas o guías turísticas (impresas) & 871 & 44,5 \\
Oficinas de información turística (Infotur) & 638 & 32,6 \\
Aplicaciones de teléfonos móviles (mapas, guías, otras) & 911 & 46,6 \\
Personal que le brinda servicios turísticos & 978 & 50,0 \\
Otra vía & 32 & 1,6 \\
\hline
\end{tabular}

Nota: Elaboración propia a partir de los cuestionarios aplicados.

La última variable comprendida dentro del cuestionario aplicado se refería a las vías utilizadas por los clientes en la obtención de la información durante la realización del viaje (ya en el destino). A ello estaba destinada la pregunta 6 del cuestionario que también era de selección múltiple (ver tabla 4). Las opciones más seleccionadas son "Preguntando en la calle" y "Personal que brinda servicios turísticos", esto demuestra la importancia que tienen tanto la población en general como el personal de las entidades turísticas como infomediarios. También son muy utilizados para obtener información durante la realización del viaje las "Aplicaciones de teléfonos móviles" y los "Mapas y guías impresas".

Los encuestados que seleccionaron la utilización de otra vía para informarse durante su estancia en el destino, señalan dentro de ellas: Internet (redes sociales, páginas sobre Cuba, entre otras), experiencia personal, consultas en su embajada y noticias. Igualmente, se preguntó sobre las informaciones no encontradas (pregunta abierta \# 7 en el cuestionario aplicado) y fueron señaladas las siguientes: funcionamiento preciso de la $\mathrm{Wi}$ $\mathrm{Fi}$, algunas direcciones específicas, mapas sobre La Habana y otros específicamente sobre las redes de transporte y restaurantes con requerimientos específicos (para vegetarianos, baratos, etc.).

$\mathrm{Al}$ analizar esta variable relacionada con la manera en que los visitantes organizan su viaje (ver figura 6), se puede observar que: 1- los que organizan el viaje de forma independiente, así como los que organizan el viaje utilizando otra manera distinta a las propuestas son los que más utilizan a los amigos y familiares y la opción de preguntar en la calle para obtener información durante la realización de su viaje. 2- Los que organizan su viaje o parte de este a través de una agencia de viaje son los que más utilizan los guías turísticos y los agentes de viajes para informarse en el destino. 3- Los visitantes, sin importar la manera en que organizaron su viaje, usan de similar forma los mapas y guías impresas, las oficinas de información turística, las aplicaciones de teléfonos móviles y la información brindada por el personal de contacto en las entidades turísticas. Los que organizaron su viaje de otra manera distinta a las mencionadas coinciden con los que más 
utilizan otra vía para informarse en la realización del viaje y, como se explicó en el análisis de la variable anterior, se trata de personas que su viaje fue organizado por escuelas, iglesias y centros de trabajo, y ellos mismos le brindan la información durante la organización y realización del viaje.

Si se analiza el comportamiento de la variable con relación al sexo se puede percibir que el comportamiento es casi idéntico entre ambos grupos (femenino y masculino). Empero, nuevamente aparecen diferencias significativas en el comportamiento presentado por los grupos etarios (ver figura 7). Más del 50\% de los menores de 31 años preguntan en la calle para obtener la información requerida durante su viaje, asimismo más del $50 \%$ de los mayores de 60 años utilizan a los guías turísticos y los agentes de viajes. Es muy llamativo el hecho de que el $65 \%$ de los menores de 21 años utilicen las aplicaciones de teléfonos móviles y solamente el 12\% de los mayores de 70 años lo hagan. Por último, señalar que entre el $40 \%$ y el $50 \%$ de los encuestados utilizan las guías y mapas impresos sin mucha determinación en la edad y que los más jóvenes son los que más utilizan el personal de contacto para obtener información durante el viaje.

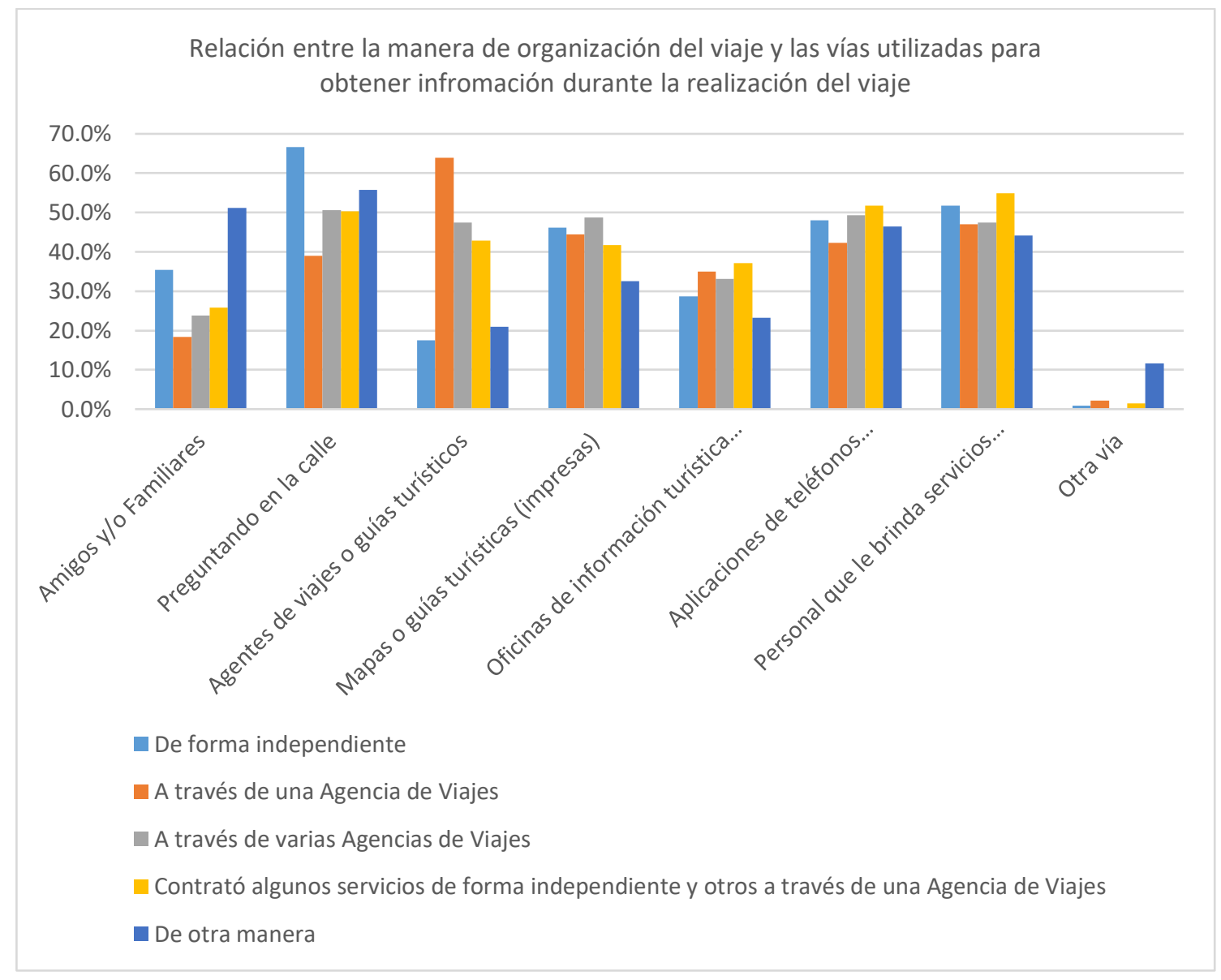

Figura 6: Relación entre la manera en que los visitantes organizan su viaje y las vías que utilizan para obtener la información necesaria durante la realización del mismo.

Elaboración propia a partir de los cuestionarios aplicados. 


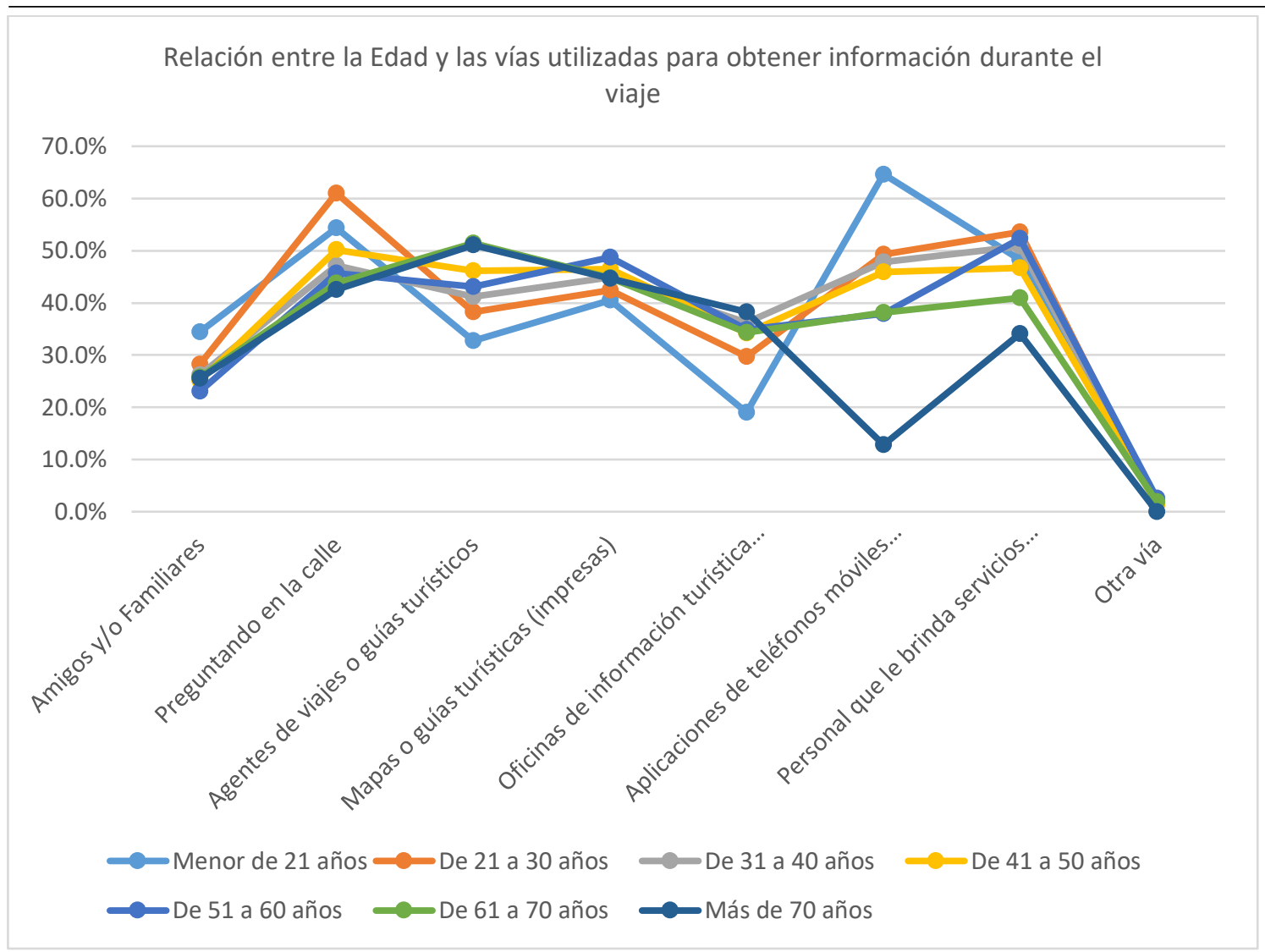

Figura 7: Relación entre la Edad y las vías que utilizan para obtener la información necesaria durante la realización del viaje. Elaboración propia a partir de los cuestionarios aplicados.

\section{Conclusiones}

A partir de los resultados presentados anteriormente se pueden arribar a las siguientes conclusiones:

- Los gestores del destino y específicamente los que se ocupan de gestionar el Sistema de Información al Visitante deben monitorear y tratar de influir en la información que los infomediarios brindan a los visitantes.

- Los infomediarios entrevistados confirmaron que los clientes que son atendidos por ellos solicitan información adicional sobre el destino que no está directamente relacionada con el negocio que atienden.

- Los infomediarios utilizan diversas fuentes para obtener la información que le brindan a los visitantes, pero dentro de ellas se destacan las aplicaciones para teléfonos móviles.

- Dentro de las vías más utilizadas por los visitantes del destino La Habana para conocer sobre la existencia de Cuba y para informarse en la organización de su viaje, se encuentran los Amigos y Familiares y las Páginas Webs sobre Cuba.

- Las vías más utilizadas para informarse durante la realización del viaje son "Preguntando en la calle" y "Preguntando al personal que brinda servicios 
turísticos. Esto demuestra la relevancia que tienen los infomediarios turísticos en la transmisión de información el destino.

- Los modos en que los clientes se informan durante la organización y realización del viaje están determinados en gran medida por la vía que utilizaron para organizar su viaje.

- Los modos en que los clientes saben sobre la existencia del destino, así como las vías que utilizan para obtener información en la organización y realización del viaje se comporta de distintas maneras en los diferentes grupos etarios.

\section{Referencias bibliográficas}

Castellanos, C. (2014). Flujo de información destino - cliente a través de los alojamientos privados. El caso de Plaza de la Revolución (Trabajo de Diploma no publicado). Universidad de La Habana, Cuba.

Manzano, D. (2016). Flujo de información destino-cliente a través de los transportistas privados de autos clásicos (Trabajo de Diploma no publicado). Universidad de La Habana, Cuba.

Martín, R. y Abreu, D. (2013). El flujo de información turística destino - cliente. Manuscrito no publicado, Centro de Estudios Turísticos, Facultad de Turismo, Universidad de La Habana, Cuba.

Martín, R. (2015). Principios, organización y práctica del turismo. Segunda parte ( $1^{\mathrm{a}} \mathrm{ed}$.). La Habana: Editorial Universitaria Félix Varela.

MINTUR (Ministerio de Turismo de la República de Cuba) (2019). Organigrama del ministerio. Recuperado de https://www.mintur.gob.cu/organigrama/

ONE (Oficina Nacional de Estadísticas de la República de Cuba) (2011). Turismo en cifras: Cuba 2010. Recuperado de https://studylib.es/doc/8650147/turismo-encifras.-cuba-2010---oficina-nacional-de-estad\%C3\%AD...

ONEI (Oficina Nacional de Estadísticas e Información de la República de Cuba) (2019). Turismo internacional: indicadores seleccionados: enero - junio 2019. Recuperado de http://www.onei.gob.cu/node/14278

Oficina Nacional de Información Turística (2014). Manual de Gestión de la Comunicación. Manuscrito no publicado, Oficina Nacional de Información Turística, Ministerio de Turismo de la República de Cuba.

Pardo, J. (2009). Sistema de información sobre la demanda turística en Ciudad de La Habana (Tesis de Maestría no publicada). Universidad de La Habana, Cuba. 
Valdés, L., Martín, R., Aza, R., Del Valle, E., y Delgado, A. (Coords.) (2011). La demanda turística hacia La Habana. Implementación adaptada del Sistema de Información Turística de Asturias. Gijón: Ediciones de la Universidad de Oviedo.

\section{Ciencia Digital}




\section{PARA CITAR EL ARTÍCULO INDEXADO.}

Abreu Rodríguez, D. A., Valdivia Izquierdo, S., \& Sánchez Borges, Y. (2021). Diagnóstico del estado actual del flujo de información entre el destino La Habana y los visitantes extranjeros. Explorador Digital, 5(1), 25-43. https://doi.org/10.33262/exploradordigital.v5i1.1488

\section{【 Ciencia}

El artículo que se publica es de exclusiva responsabilidad de los autores y no necesariamente reflejan el pensamiento de la Revista Explorador Digital.

El artículo queda en propiedad de la revista y, por tanto, su publicación parcial y/o total en otro medio tiene que ser autorizado por el director de la Revista Explorador Digital.
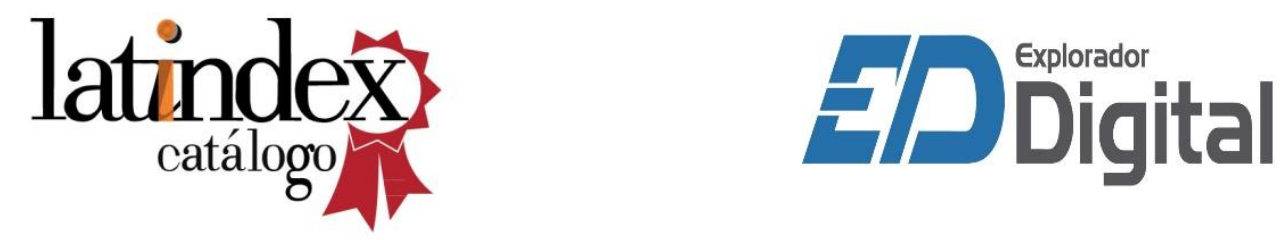